\title{
MINIMIZING USER AND OPERATOR COSTS OF SINGLE LINE BUS SERVICE USING OPERATIONAL STRATEGIES
}

\author{
Chunyan TANG ${ }^{1}$, Avishai (Avi) CEDER ${ }^{2}$, Shengchuan $\mathrm{ZHAO}^{3}$ \\ ${ }^{1}$ College of Transportation and Engineering, Dalian Maritime University, China \\ ${ }^{2}$ Transportation Research Centre, Faculty of Engineering, University of Auckland, New Zealand \\ ${ }^{3}$ School of Transportation and Logistics, Dalian University of Technology, China
}

Received 18 February 2017; revised 6 July 2017; accepted 24 November 2017

\begin{abstract}
This work presents a methodology for minimizing costs involved in the operation of a single line bus service. The model developed is based on optimal implementation of operational strategies tailored to passenger demand for a bidirectional single bus line. As a result, the commonly used timetable for Full Route Operation (FRO) will have to change to accommodate three types of strategies: short turn, limited stop, and mixed strategy (a combination of short turn and limited stop). The use of operational strategies will better match supply and demand, and will thus improve operation efficiency. The optimization model determines which trips of the given FRO timetable will be implemented with given strategies considering the trade-offs between passenger and operator costs. Moreover, in applying the model, the availability of real time information for passengers is considered in the calculation of waiting times. The proposed model is interpreted in the context of a small example, which serves as an explanatory devise. Then, it is applied to a real life case study in Dalian, China. The results show an indication that a significant saving could be attained by the use of multiple strategies. These savings were especially observed in the reduction of operational costs involved with the saving of travel times and running empty seats.
\end{abstract}

Keywords: public transit, operational strategies, timetable, full route operation, optimization.

\section{Introduction}

Trip generation and trip attraction is closely related to land zone use. A greater number of passengers appear in zones such as shopping zones, business zones, school zones, resident zones, etc. Passenger demand distribution along an urban bi-directional bus route passing through different types of zones shows neither uniformity nor symmetry. However, in practice, full advantage is not always taken of public transit resources to meet such imbalanced travel demand distribution, due to the fact that common Full Route Operation (FRO) strategy is provided for the entire timetable. Such an operation situation could be efficiently improved by shifting some FRO bus trips to operate by other strategies. This is because the use of other strategies opens up an opportunity to further save vehicle operating time, to reduce vehicle empty seat hours, and even to decrease the number of vehicles required, while ensuring that service levels for passengers are not less than an ac- ceptable minimum value. These strategies include short turn, limited stop, deadhead, express, and zonal strategy, for which application for different beneficial conditions is illustrated, particularly by Furth and Day (1985).

Short turn strategy used by buses serves short cycles or loops on a route. Specifically, this strategy increases service frequencies on high load route segments (Furth 1987; Ceder 1989). Limited stop strategy operated by buses visits only a subset of stops on a route. This results in stops with high demand getting more service than those stops with low demand (Fu et al. 2003). A deadheading strategy defines bus transfers from one terminal where it is not needed to another where it is needed to service a required trip (Ceder, Stern 1981). Apparently, this strategy benefits routes with imbalanced demand in both directions because of improving service levels for the direction with high demand. Additionally, in some route segments, a

${ }^{*}$ Corresponding author. E-mail: a.ceder@auckland.ac.nz 
short turn strategy may be advantageous, while in the next segment either limited stop or deadheading may be better. In these cases, a mixed strategy (Cortés et al. 2011) and multiple strategies (Tang et al. 2016) are worth exploring.

\section{Literature review}

A considerable amount of literature focuses on determination of operational strategies and their associated frequencies using mathematical programming methods for intermediate level planning of bus operations. Delle Site and Filippi (1998) proposed an optimization model to determine frequencies of FRO and short turn for a given corridor operation. This model produces trade-offs between users' and operator's costs. Ulusoy et al. (2010) introduced express strategy into the FRO and short turn strategies, using a logit based model to estimate ridership with these strategies. Considering the effect of using operational strategies on passenger path choices and transfer behaviour, Chien et al. (2010b) and Qu et al. (2016) further investigated all-stop and stop-skipping service patterns for reducing the total social cost. For corridors with imbalanced demand between directions, an integrated strategy of short turn and deadheading is presented in the study by Cortés et al. (2011). By optimizing frequencies of deadheading and short turn with and outside the high demand zone, a cost-efficient operation is constructed in their study. Moreover, Chiraphadhanakul and Barnhart (2013) introduced a limited stop strategy to be operated simultaneously with an existing FRO strategy. Replacing additional trips provided by optimally reassigning some bus trips is applied to modify a given bus service on a particular corridor. Finally, a mixed integer nonlinear model is formulated to determine the limited stop strategy and its associated frequency.

In order to accommodate the more complex demand features of a bus corridor, Leiva et al. (2010) proposed an optimization approach to examine which of given strategies consisting of limited stop, normal, and express, should be provided at what frequencies with a given origin-destination demand matrix. Based on this model proposed by Leiva et al. (2010), Larrain et al. (2010) considered different characteristics of demand on a bus corridor, including the base load profile shape, the scale of demand, and the demand imbalance between outbound and inbound directions. As a result, strategies and their associated frequencies are determined to accommodate these demand conditions. In recent studies, a genetic algorithm (Ulusoy, Chien 2015) and heuristics (Larrain et al. 2015) approaches have been applied to deal with a frequency optimization problem in designing operational strategies.

Although extensive research has been conducted to use operational strategies for better matching between demand and supply, the implementation of new operational strategies based on the methods proposed above must result in a new timetable. This benefits new route operations, while for current operation routes, a changeable timetable might frustrate passengers and reduce attractiveness of public transit. Thus, there is need to eliminate, as much as possible, this frustration of passengers and simultaneously improve the efficiency of current route operation. In earlier attempts, Ceder, Stern (1981) and Furth (1987) focused on shifting a few FRO bus trips from a given timetable to operate using short turn strategy or by inserting deadheading trips can save the number of vehicles required. In order to reduce an adverse effect for some passengers who want to board or alight at skipped stops, they try to minimize total waiting time based on the constraint without increasing the resulting fleet size. This approach may be useful for shifting bus trips to operate by short turn strategy because it only increases waiting time for passengers at the stops not served (skipped). Nevertheless, it will be difficult to get a better trade-off between user and operator costs for shifting bus trips to use limited stop strategy. This is because it will increase waiting time for passengers boarding and alighting at skipped stops, but it also reduces in-vehicle time for passengers passing through those skipped stops.

In this work, we overcome this issue, by a development of an optimization model with the objective of minimizing total passenger and operator costs using multiple operational strategies based on a given timetable. To the authors' knowledge, the present study provides for the first time in the literature the construction of multiple strategies tailored to the demand distribution based on a given timetable. The formulation of cost components in the developed model is mainly related to the operational characteristics in practice for a given timetable; this is different from what was developed by previous studies without a given timetable (Liu et al. 2013; Chen et al. 2015; Chien et al. 2010a; Zhao, Chien 2015).

In addition, in the proposed model, we are able to consider a saving rate of waiting time because public transport information in real time is available for passengers. Real time information displays are not a recent phenomenon, and were first introduced in Chicago in the early 1970s with vehicles using automatic vehicle location (Dziekan, Kottenhoff 2007). Today, real time public information displayed via smartphones can provide a range of information to its users, including vehicle positioning, travel time and speed, passengers on board, and different route/mode options. This can assist customers in selecting their bus routes between their current locations and destinations (Cats, Loutos 2016). A study by Watkins et al. (2011) found that when public transit users were provided with real time information, waiting time was reportedly reduced by two minutes. Thus reduced waiting time is made possible by timing arrival time at the stops.

In what follows, a set of strategies are presented which aims to improve operation system efficiency. Section 2 develops an optimization model for constructing operational strategies based on a given timetable. A detailed exam- 
ple is used to illustrate the proposed model in Section 3. Section 4 applies the model to a real life case study from Dalian, China. Finally, the main findings are summarized, and extensions of this approach are proposed in the last section.

\section{Problem formulation}

This section proposed a model to construct operational strategies tailored to passenger demand for a bi-direction single bus line, based on a given timetable. This proposed model focuses on shifting some FRO bus trips to operate by other strategies, including short turn, limited stop, and mixed strategy (a combination of short turn and limited stop), as shown in Figure 1. It relies on the following input data: (1) a complete timetable of all route time points; (2) passenger demand for each operation period. In this work, we divide the operation time into several periods, and consider each hour as a single period. Bus arrivals at stops are assumed to match the timetable. In addition it is assumed that passengers would not transfer between vehicles with different operational strategies on the single bus line. The short turn trips, as is defined by Ceder (2007, 2015) are initiated beyond the route departure point and/ or terminated before the route arrival point. This study investigates only the case of short turn trips starting beyond the route departure point. The feasible short turn points are all intermediate stops along a bus route where the vehicle can turn back without interfering with the traffic flow.

\subsection{Notations}

In order to formulate the methodology as a programming model, the parameters and variables are defined as follows:

\section{Indices and sets:}

$i$ - index of stops on the bus line in one direction, $i=1,2, \ldots, N$

$k$ - index of bus trips for a given timetable;

$l$ - index of turning points along the bus line, $l=2$, $3, \ldots, N-1$;

$E$ - set of end bus trips of all operation periods in both directions of the bus line;

$S$ - set of start bus trips of all operation periods in both directions of the bus line;

\section{Parameters:}

$c_{1}$ - unit time value associated with passenger waiting time [\$/hour];

$c_{2}$ - unit time value associated with passenger in-vehicle time [\$/hour];

$c_{3}$ - unit time value associated with bus travel time [\$/ hour];

$c_{4}$ - unit time value associated with vehicle empty seat hours [\$/hour];

$H_{k 0}$ - given headway of bus trip $k$ obtained by a given timetable [hour];

$K$ - the given number of bus trips for a single-line bus operation;

$N$ - the number of stops of the bus line in one direction;

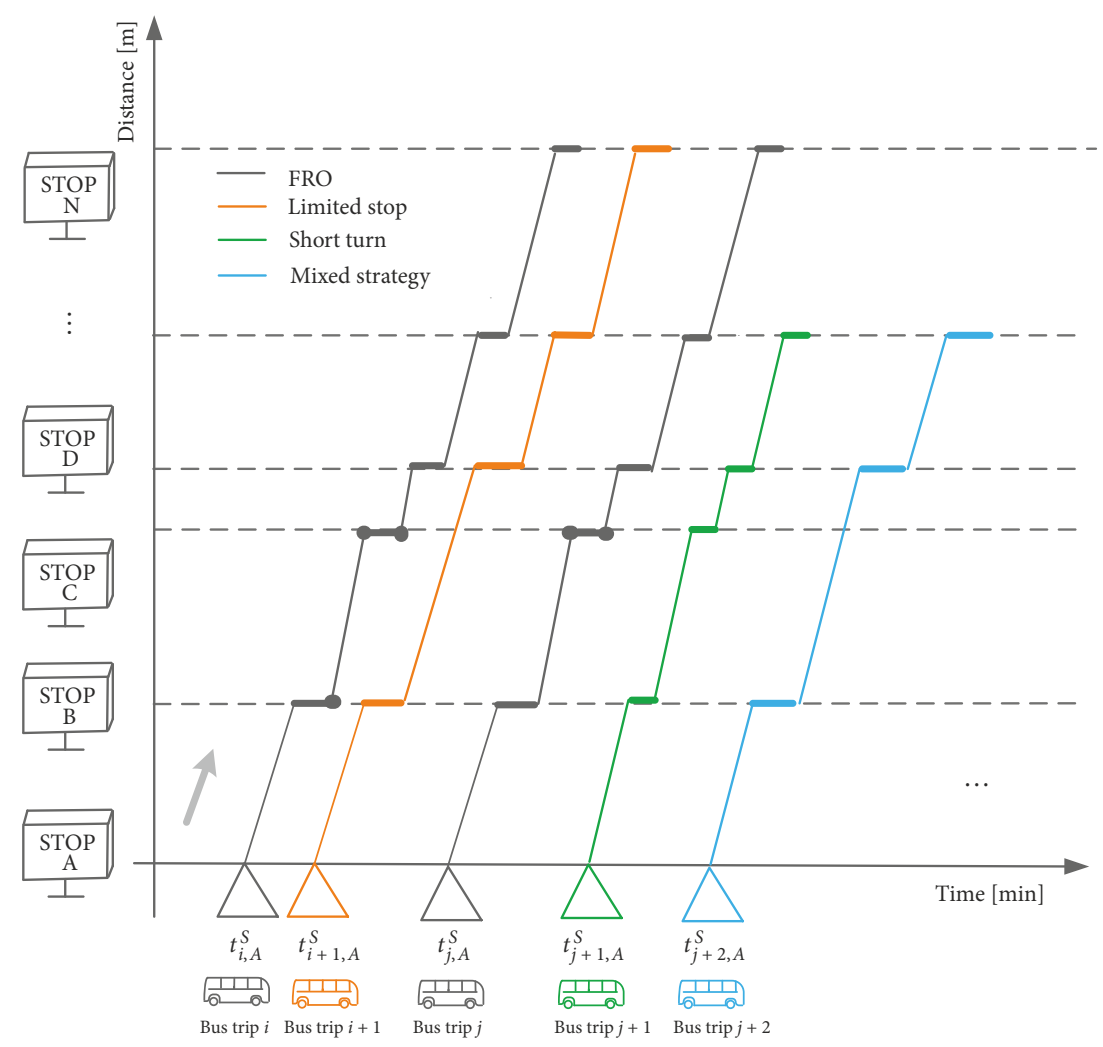

Figure 1. Space-time diagram of operational strategies of a bus single line at given operation period 
$N B_{i, j}$ - the number of stops between stop $i$ and stop $j$;

$q$ - desired occupancy of bus [passengers/vehicle];

$R_{i}-$ the required number of bus trips serving stop $i$;

$R T_{i, j}$ - original travel time between stop $i$ and stop $j$ [hour];

$t$ - average time saving per skipped stop [hour];

$\theta$ - saving rate of passenger waiting time;

$\lambda_{i, j}^{h}$ - average passenger arrival rate at stop $i$ whose destination is stop $j$ during operation period $h$ [passengers/hour];

$\eta_{i, j}^{p}$ - taking a value of 1 if stop $p$ is located between stop $i$ (included) and stop $j$, and 0 otherwise.

\section{Variables:}

$H_{k, i}$ - headway of bus trip $k$ at stop $i$ [hour];

headway change for passengers on bus trip $k$

$\Delta H_{i, j}^{k}$ - boarding at stop $i$ and alighting at stop $j$ based on the former bus trip $k-1$ operating performance at stop $i$ [hour];

$L D_{p}$ - bus empty seat hours on the route segment between stop $p$ and stop $p+1$ [hour];

$N A_{i, j}^{k}$ - the number of stops visited by bus trip $k$ between stop $i$ and stop $j$;

$\Delta R T_{i, j}^{k}$ - travel time of bus trip $k$ between stop $i$ and stop $j$ [hour];

$S C$ - total costs of passengers and operator [\$];

$T R_{k}$ - travel time of bus trip $k$ [hour];

$W T_{k, i}$ - waiting time of passengers on bus trip $k$ boarding at stop $i$ [hour];

$V T_{k, i}$ - in-vehicle time of passengers on bus trip $k$ boarding at stop $i$ [hour];

$V_{i, j}^{k}$ - the number of passengers on bus trip $k$ boarding at stop $i$ and alighting at stop $j$;

$\delta_{k, i}$ - binary variable that equals 1 if bus trip $k$ serves stop $i$, and 0 otherwise.

\subsection{Model with operational strategies}

To some stops with low passenger boarding and alighting, some bus trips are allowed to skip these stops. As a result, this must be able to improve efficiency of bus operation, such as savings in vehicle travel time, in empty seat hours, and even in the number of vehicles required. Additionally, it benefits for passengers whose origins and destinations are served, and some of their intermediate stops are skipped. However, it also results in the adverse side for those passengers who want to board or alight at skipped stops. In order to overcome this issue, a model is developed to determine which of given FRO bus trips to skip those stops with low demand, while taking the tradeoffs between passengers' cost and operator's cost into account. The objective function of this model consists of the equivalent total costs of passenger waiting time, passenger in-vehicle time, bus travel time, and vehicle empty seat hours as follows:

$$
S C=c_{1} \sum_{k=2}^{K} \sum_{i=1}^{N}(1-\theta) \cdot W T_{k, i}+
$$

$$
c_{2} \sum_{k=2}^{K} \sum_{i=1}^{N} V T_{k, i}+c_{3} \sum_{k=2}^{K} T R_{k}+c_{4} \sum_{i=1}^{N-1} L D_{i} .
$$

Because time values associated with passengers and operator are not of equal importance, these terms are converted to common units of cost in dollars with weighting factors $c_{1}, c_{2}, c_{3}$, and $c_{4}$ for passenger waiting time, passenger in-vehicle time, bus travel time, and bus empty seat hours, respectively. $\theta$ represents a saving rate of waiting time because bus arrival time in real time is available for passengers, and thus allows for reducing waiting time by timing the arrival time at the stops. A greater value indicates that passengers know well about bus arrival time in real time, and thus obtain more savings in waiting time.

\section{Waiting time}

The waiting time at a stop depends on the headway at this stop. When a passenger's origin or destination is skipped by a bus, the passenger will not board this bus and wait for the next bus. The waiting time $W T_{k, i}$ for passengers using vehicle trip $k$ at stop $i$ is expressed as follows:

$$
\begin{aligned}
& W T_{k, i}=\sum_{j=1}^{N} V_{i, j}^{k} \cdot \frac{\Delta H_{i, j}^{k}}{2} ; \\
& V_{i, j}^{k}=\delta_{k, i} \cdot \delta_{k, j} \cdot \lambda_{i, j}^{h} \cdot \Delta H_{i, j}^{k} ; \\
& \Delta H_{i, j}^{k}=H_{k, i}+H_{k-1, i} \cdot\left(1-\delta_{k-1, i} \delta_{k-1, j}\right) .
\end{aligned}
$$

Equation (4) represents the headway change for passengers using bus trip $k$ to board at stop $i$ and alight at stop $j$ that depends on the headway of bus trip $k$ and performance of the former bus trip $k-1$. If passengers can board bus trip $k-1$ at stop $i$ and alight at stop $j$, the headway change equals the headway of bus trip $k$ at stop $i$. However, if not, the headway change is a sum of the headway of bus trip $k$ and the headway of bus trip $k-1$ at stop $i$.

The headway of a bus trip at a stop depends on the former bus trip operating performance and the bus trip itself operating performance at the former stop. The headway will increase if the former bus trip skips the former bus stop, while it will reduce if the bus trip itself skips the former stop. The headway of a bus trip $k$ at stop $i$ is formulated as:

$$
H_{k, i}=\left\{\begin{array}{l}
H_{k 0}, \text { if } \sum_{p=1}^{i-1} \delta_{k, p}=0 \text { or } \sum_{p=1}^{i-1} \delta_{k-1, p}=0 \text { and } \delta_{k-1, i}=1 \\
H_{k 0}+H_{k-10}, \text { if } \sum_{p=1}^{i-1} \delta_{k-1, p}=0 \text { and } \delta_{k-1, i}=0 \\
H_{k, i-1}+\left(1-\delta_{k-1, i-1}\right) \cdot t-\left(1-\delta_{k, i-1}\right) \cdot t, \text { otherwise. }
\end{array}\right.
$$

In the Equation (5), the headway is computed by the first expression if bus trip $k$ starts from the turning stop $l$ along the route and $i \leq l$; by the second expression if bus trip $k-1$ starts from the turning stop $l$ and $i=l$; and by the third expression if bus trip $k-1$ starts from the turning stop $l$ and $i<l$. 


\section{In-vehicle time}

The in-vehicle time that passengers experience on buses, $V T_{k, i}$ for passengers boarding bus trip $k$ at stop $i$, is expressed as:

$$
\begin{aligned}
& V T_{k, i}=\sum_{j=1}^{N} V_{i, j}^{k} \cdot \Delta R T_{i, j}^{k} ; \\
& \Delta R T_{i, j}^{k}=\left(R T_{i, j}-\left(N B_{i, j}-N A_{i, j}^{k}\right) \cdot t\right) .
\end{aligned}
$$

\section{Bus travel time}

The travel time of a bus trip depends on operating performances. When a bus trip operates by short turn or limited stop strategies, it performs shorter travel time compared with operating FRO strategy. In this study, the operating time $T R_{k}$ for bus trip $k$ is formulated as:

$$
T R_{k}=\left\{\begin{array}{l}
R T_{l, N}-\left(N B_{l, N}-\sum_{i=l}^{N} \delta_{k, i}\right) \cdot t, \text { if } \sum_{i=1}^{l-1} \delta_{k, i}=0 ; \\
R T_{1, N}-\left(N B_{1, N}-\sum_{i=1}^{N} \delta_{k, i}\right) \cdot t, \text { otherwise. }
\end{array}\right.
$$

In the Equation (8), the bus travel time is computed by the first expression if bus trip $k$ starts at stop $l$ along the route and by the second expression otherwise.

\section{Vehicle empty seat hours}

Vehicle empty seat hours are involved in the provided number of bus trips and the required number of bus trips for a given route segment between stop $p$ and stop $p+1$. When the provided number is more than the required number, it results in empty seats. $L D_{p}$ represents the vehicle empty seat hours between stop $p$ and stop $p+1$, which is developed as:

$$
L D_{p}=\sum_{k=2}^{K}\left(q-\sum_{i=1}^{N} \sum_{j=1}^{N} \eta_{i, j}^{p} \cdot V_{i, j}^{k}\right) \cdot R T_{p, p+1} .
$$

In summary, combining Equations (2)-(9) with Equation (1), the final optimization model for a direction of the route bus during a single operation period $h$ is formulated as follows:

$$
\begin{aligned}
& \min S C=c_{1} \cdot \sum_{k=2}^{K} \sum_{i=1}^{N} \sum_{j=1}^{N}\left((1-\theta) \cdot \delta_{k, i} \cdot \delta_{k, j} \cdot \lambda_{i, j}^{h} \times\right. \\
& \left.\frac{\left.\left(H_{k, i}+H_{k-1, i} \cdot\left(1-\delta_{k-1, i} \delta_{k-1, j}\right)\right)^{2}\right)}{2}\right) \\
& c_{2} \cdot \sum_{k=2}^{K} \sum_{i=1}^{N} \sum_{j=2}^{N}\left(\delta_{k, i} \cdot \delta_{k, j} \cdot \lambda_{i, j}^{h} \times\right. \\
& \left(H_{k, i}+H_{k-1, i} \cdot\left(1-\delta_{k-1, i} \cdot \delta_{k-1, j}\right)\right) \times \\
& \left.\left(R T_{i, j}-\left(N B_{i, j}-N A_{i, j}^{k}\right) \cdot t\right)\right)+ \\
& c_{3} \cdot \sum_{k=2}^{K} T R_{k}+c_{4} \times
\end{aligned}
$$

$$
\begin{aligned}
& \sum_{p=1}^{N-1} \sum_{k=2}^{K}\left(\left(q-\sum_{i=1}^{N} \sum_{j=1}^{N} \eta_{i, j}^{p} \cdot \delta_{k, i} \cdot \delta_{k, j} \cdot \lambda_{i, j}^{h} \times\right.\right. \\
& \left.\left.\left(H_{k, i}+H_{k-1, i} \cdot\left(1-\delta_{k-1, i} \cdot \delta_{k-1, j}\right)\right)\right) \cdot R T_{p, p+1}\right)
\end{aligned}
$$

subject to:

$$
N A_{i, j}^{k}=\left\{\begin{array}{l}
\sum_{p=i}^{j} \delta_{k, p}, \text { if } i \leq j \\
\sum_{p=j}^{i} \delta_{k, p}, \text { if } i>j
\end{array}\right.
$$

$k=2, \ldots, K$;

$\delta_{k, i} \in\{0,1\}, k=1, \ldots, K, i=1, \ldots, N$;

$\delta_{k, i}=1$, if $k \in S$ or $k \in E, i=1, \ldots, N$;

$\sum_{k=2}^{K} \delta_{k, i} \geq R_{i}, i=1, \ldots, N$

$\delta_{k, i} \cdot \delta_{k, j}+\delta_{k+1, i} \cdot \delta_{k+1, j} \geq 1, k=1, \ldots, K-1 ;$

$H_{k, i} \geq 0, k=1, \ldots, K, i=1, \ldots, N$;

$H_{k, 1}=H_{k 0}, k=1, \ldots, K$.

In this model, Constraint (11) calculates the number of stops that bus trips serve between two stops. Constraint (12) shows whether bus trips to serve a stop; equal 1 if bus trips serve a stop and 0 otherwise. Constraint (13) guarantees the first and end bus trips of operation period serve all stops in both directions along the given bus line. Constraint (14) shows that the number of serving trips is more than the desired number of bus trips at each stop in order to maintain a comfortable average passenger load and eliminate the number of standees (if any) which exceed an average, acceptable level. Constraint (15) ensures that all passengers can board at least one of two consecutive buses. Constraint (16) is set as an operational constraint in order to avoid vehicle bunching. The initial headways of bus trips are given in Constraint (17).

The model developed is a nonlinear integer model. It can be handled by the Outer Approximation with both Equality Relaxation and Augmented Penalty (OA/ER/AP) algorithm of Viswanathan and Grossmann (1990) and Branch and Bound (B-B) algorithm. OA/ER/AR algorithm is readily available in the DICOPT solver of GAMS (Brooke et al. 2005; Grossmann et al. 2018). The B-B algorithm is coded in LINGO or the SBB solver of GAMS. Some heuristic methods, coded using MATLAB, can also be used to solve this model, such as genetic algorithm and ant colony algorithm.

\section{Example}

A numerical example is used to illustrate the operational strategies problem and to better understand the proposed model. Figure 2 presents a bi-directional bus line with five 


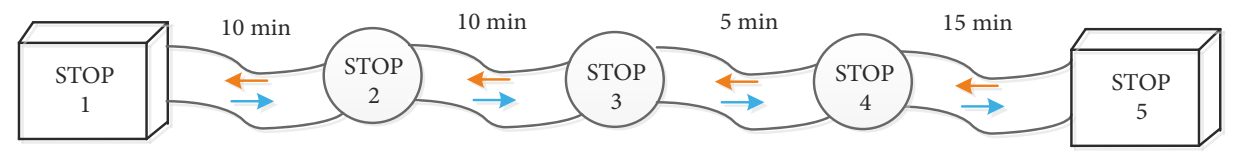

Figure 2. An example of bi-directional bus line

stops in each direction. Travel time between two consecutive stops is shown in Figure 2. The operation time periods are divided into two periods, 7:00...8:00, and 8:00...9:00, denoted as $h_{1}$ and $h_{2}$ respectively. The average passenger demand arrival rate at the origin stop whose destination is another stop is displayed in Table 1 . The complete FRO timetable is exhibited in Table 2. The minimum frequency is 2 buses per hour. The desired occupancy on a bus is set for 50 seats. When a bus skips a stop, it will save 5 minutes for travel time.

Table 1. Passenger average demand rate between origindestination (O-D) [passengers/min]

\begin{tabular}{|c|c|c|c|c|c|}
\hline $\mathrm{O} \quad \mathrm{D}$ & 1 & 2 & 3 & 4 & 5 \\
\hline \multicolumn{6}{|c|}{ 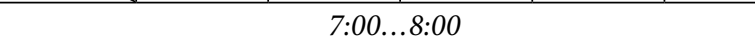 } \\
\hline 1 & 0 & 2.00 & 1.00 & 0.49 & 0.67 \\
\hline 2 & 3.15 & 0 & 0.67 & 0.25 & 0.25 \\
\hline 3 & 0.40 & 0.10 & 0 & 1.6 & 0.90 \\
\hline 4 & 0.25 & 0.25 & 0.83 & 0 & 4.01 \\
\hline 5 & 0.50 & 0.50 & 0.67 & 4.33 & 0 \\
\hline \multicolumn{6}{|c|}{$8: 00 \ldots 9: 00$} \\
\hline $\mathrm{O} D$ & 1 & 2 & 3 & 4 & 5 \\
\hline 1 & 0 & 2.60 & 0.40 & 0.50 & 0.50 \\
\hline 2 & 2.86 & 0 & 0.85 & 0.40 & 0.35 \\
\hline 3 & 1.00 & 0.85 & 0 & 0.15 & 0.10 \\
\hline 4 & 0.33 & 0.33 & 0.12 & 0 & 3.05 \\
\hline 5 & 1.45 & 0.25 & 0.85 & 3.00 & 0 \\
\hline
\end{tabular}

First, according to observed passenger demand rates during operation periods (Table 1), passenger load profiles in each direction at given operation periods can be generated, as shown in Figure 3.

Based on generated load profiles, the number of bus trips required for each stop can be determined. For instance, the required number of bus trips serving stop 3 in Figure 3, is calculated as:

$$
R_{3}=\max \left(\frac{200}{q}, \frac{250}{q}, 2\right) \text {. }
$$

In the above equation, the first term represents the required number of bus trip arrival at stop 3 , the second term for the required number of bus trip departures, and the last one for the minimum number of bus trips required. The desired occupancy on a bus is set for $q=50$ passengers in this small example.

Second, run the model with the objective of minimizing the total costs, including waiting time and in-vehicle time for passengers, as well as bus travel time and empty seat hours for operator. As shown in Figure 3a, calculate waiting time and in-vehicle time for passengers boarding bus trip 6 at the stop 3 as follows:

$$
\begin{aligned}
& W T_{6,3}=\delta_{6,3} \cdot \delta_{6,4} \cdot \lambda_{3,4}^{h 1} \times \\
& \frac{\left(H_{6,3}+H_{5,3} \cdot\left(1-\delta_{5,3} \cdot \delta_{5,4}\right)\right)^{2}}{2}+\delta_{6,3} \cdot \delta_{6,5} \cdot \lambda_{3,5}^{h 1} \times \\
& \frac{\left(H_{6,3}+H_{5,3} \cdot\left(1-\delta_{5,3} \cdot \delta_{5,5}\right)\right)^{2}}{2} ;
\end{aligned}
$$

\begin{tabular}{|c|c|c|c|c|c|c|c|c|c|c|c|c|c|}
\hline & & \multicolumn{5}{|c|}{ Direction 1} & \multicolumn{5}{|c|}{ Direction 2} & \multirow[b]{2}{*}{$\begin{array}{l}\text { Bus } \\
\text { trip } \\
\text { No. }\end{array}$} & \multirow[b]{2}{*}{ Time period } \\
\hline \multicolumn{2}{|c|}{ Time } & 1 & 2 & 3 & 4 & 5 & 5 & 4 & 3 & 2 & 1 & & \\
\hline \multirow{7}{*}{$\begin{array}{l}7: 00 \ldots 8: 00 \\
\text { (at stop 2) }\end{array}$} & 1 & $7: 00$ & $7: 10$ & $7: 20$ & $7: 25$ & $7: 40$ & $7: 00$ & $7: 15$ & $7: 20$ & $7: 30$ & $7: 40$ & 8 & \multirow{6}{*}{$\begin{array}{l}7: 00 \ldots 8: 00 \\
\text { (at stop 3) }\end{array}$} \\
\hline & 2 & $7: 10$ & $7: 20$ & $7: 30$ & $7: 35$ & $7: 50$ & $7: 15$ & $7: 30$ & $7: 35$ & $7: 45$ & $7: 55$ & 9 & \\
\hline & 3 & $7: 25$ & $7: 35$ & $7: 45$ & $7: 50$ & $8: 05$ & $7: 20$ & $7: 35$ & $7: 40$ & $7: 50$ & $8: 00$ & 10 & \\
\hline & 4 & $7: 35$ & $7: 45$ & $7: 55$ & $8: 00$ & $8: 15$ & $7: 25$ & $7: 40$ & $7: 45$ & $7: 55$ & $8: 05$ & 11 & \\
\hline & 5 & $7: 40$ & $7: 50$ & $8: 00$ & 8:05 & $8: 20$ & $7: 30$ & $7: 45$ & $7: 50$ & 8:00 & $8: 10$ & 12 & \\
\hline & 6 & $7: 45$ & $7: 55$ & $8: 05$ & $8: 10$ & $8: 25$ & $7: 40$ & $7: 55$ & $8: 00$ & $8: 10$ & $8: 20$ & 13 & \\
\hline & 7 & $7: 50$ & $8: 00$ & $8: 10$ & $8: 15$ & $8: 30$ & $7: 50$ & $8: 05$ & $8: 10$ & $8: 20$ & $8: 30$ & 19 & \multirow{6}{*}{$\begin{array}{l}8: 00 \ldots 9: 00 \\
\text { (at stop 3) }\end{array}$} \\
\hline \multirow{5}{*}{$\begin{array}{l}8: 00 \ldots 9: 00 \\
\text { (at stop 2) }\end{array}$} & 14 & 8:00 & $8: 10$ & $8: 20$ & $8: 25$ & $8: 40$ & $8: 05$ & $8: 20$ & $8: 25$ & $8: 35$ & $8: 45$ & 20 & \\
\hline & 15 & $8: 15$ & $8: 25$ & $8: 35$ & $8: 40$ & $8: 55$ & $8: 15$ & $8: 30$ & $8: 35$ & $8: 45$ & $8: 55$ & 21 & \\
\hline & 16 & $8: 25$ & $8: 35$ & $8: 45$ & $8: 50$ & 9:05 & $8: 20$ & $8: 35$ & $8: 40$ & $8: 50$ & 9:00 & 22 & \\
\hline & 17 & $8: 40$ & $8: 50$ & 9:00 & 9:05 & $9: 20$ & $8: 25$ & $8: 40$ & $8: 45$ & $8: 55$ & 9:05 & 23 & \\
\hline & 18 & $8: 50$ & 9:00 & $9: 10$ & $9: 15$ & $9: 30$ & $8: 35$ & $8: 50$ & $8: 55$ & $9: 05$ & $9: 15$ & 24 & \\
\hline
\end{tabular}

Table 2. Timetable during two-hour operations

Note: "at stop 2" or "at stop 3" represent the stop number at which the time period is determined. 

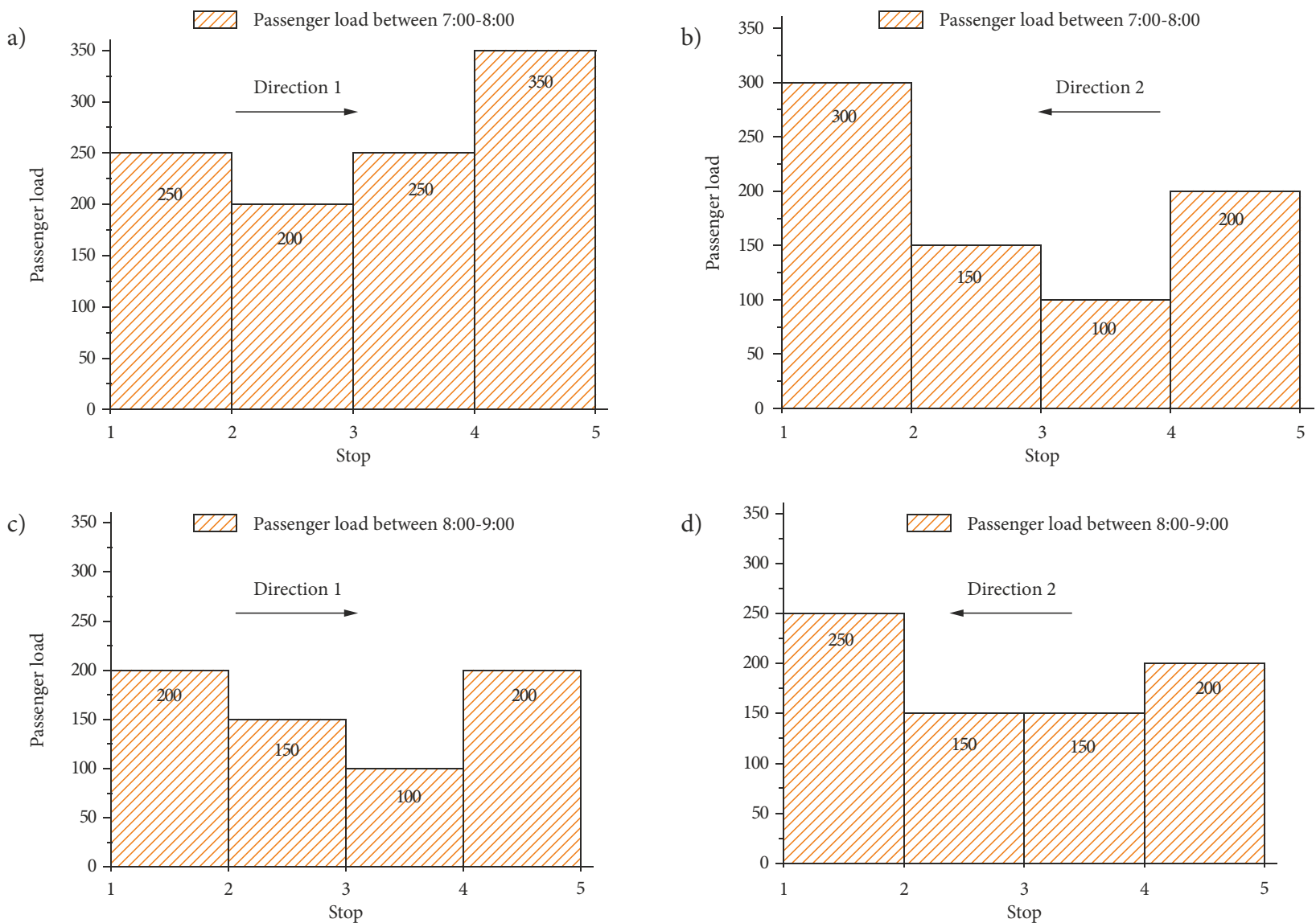

Figure 3. Load profiles on the bi-directional bus route between 7:00 ..9:00: a - 7:00 ..8:00 in direction 1; $\mathrm{b}-7: 00 \ldots 8: 00$ in direction $2 ; \mathrm{c}-8: 00 \ldots 9: 00$ in direction $1 ; \mathrm{d}-8: 00 \ldots 9: 00$ in direction 2

$$
\begin{aligned}
& V T_{6,3}=\delta_{6,3} \cdot \delta_{6,4} \cdot \lambda_{3,4} \cdot\left(H_{6,3}+H_{5,3} \cdot\left(1-\delta_{5,3} \delta_{5,4}\right)\right) \times \\
& \left(5-\left(2-\delta_{6,3}-\delta_{6,4}\right) \cdot t\right)+\delta_{6,3} \cdot \delta_{6,5} \cdot \lambda_{3,5} \times \\
& \left(H_{6,3}+H_{5,3} \cdot\left(1-\delta_{5,3} \delta_{5,5}\right)\right) \times \\
& \left(5+15-\left(3-\delta_{6,3}-\delta_{6,4}-\delta_{6,5}\right) \cdot t\right) .
\end{aligned}
$$

When a bus skips a stop, it will save $t=5$ minutes for travel time. The headway of bus trip 6 at stop 3 is updated as follows:

$$
H_{6,3}=\left\{\begin{array}{l}
H_{60}, \text { if } \sum_{p=1}^{2} \delta_{6, p}=0 \text { or } \sum_{p=1}^{2} \delta_{5, p}=0 \text { and } \delta_{5,3}=1 ; \\
H_{60}+H_{50}, \text { if } \sum_{p=1}^{2} \delta_{5, p}=0 \text { and } \delta_{5,3}=0 ; \\
H_{6,2}+\left(1-\delta_{5,2}\right) \cdot t-\left(1-\delta_{6,2}\right) \cdot t, \text { otherwise. }
\end{array}\right.
$$

When considering candidate turning point $l=3$, travel time for bus trip 6 is expressed as:

$$
T R_{6}=\left\{\begin{array}{l}
R T_{1,5}-\left(N B_{1,5}-\sum_{i=1}^{5} \delta_{6, i}\right) \cdot t, \text { if } \delta_{6,1}=1 \\
R T_{2,5}-\left(N B_{2,5}-\sum_{i=2}^{5} \delta_{6, i}\right) \cdot t, \text { if } \delta_{6,1}=0 .
\end{array}\right.
$$

Empty seat hours on the route segment between stop 3 and stop 4 in Figure $3 a$ is expressed as:

$$
\begin{gathered}
L D_{3}=\sum_{k=2}^{7}\left(\left(q-\sum_{k=2}^{7}\left(V_{1,4}^{k}+V_{1,5}^{k}+\right.\right.\right. \\
\left.\left.\left.V_{2,4}^{k}+V_{2,5}^{k}+V_{3,4}^{k}+V_{3,5}^{k}\right)\right) \cdot R T_{3,4}\right) .
\end{gathered}
$$

Based on the approach described, considering $c_{1}=$ $15 \$ /$ hour, $c_{2}=10 \$ /$ hour, $c_{3}=150 \$ /$ hour, $c_{4}=2 \$ /$ hour, and $\theta=0.5$, a model is developed to generate a timetable by shifting some FRO trips from a given timetable to operate by multiple optimal strategies in Table 3, along with the optimal topologies of strategies operated by bus trips in Figure 4.

Effects of using operational strategies on passengers and operator are presented in Table 4 . Based on the given timetable operated by FRO strategy and the generated timetable operated by multiple optimal strategies, the numbers of buses operating both timetables are calculated using Deficit-Function (DF) approach. This DF method is a highly informative, graphic person-computer interactive technique based on a step function, which is illustrated in detail by Ceder $(2007,2015)$.

As shown in Table 4, shifting some FRO bus trips to use limited stop and mixed strategy reduces the total costs of passengers and operator by $1.91 \%$, compared with all bus trips only operating FRO strategy. It may result in an increase of approximately $11.35 \%$ in total passenger wait- 
ing time cost, while bringing more savings for operating cost, such as saving $5.68 \%$ in bus travel time and $38.46 \%$ in empty seat hour cost. Additionally, it will save one bus, though this is not explicitly noted in the objective function.

In addition, the users will be compensated for the increase of efficiency, where part of the operational-cost saving will come back to the users through improving the level-of-service, such as providing better facilities, better vehicles, and better online information. Finally, the use of smartphone apps will enable the display of bus arrival time in real time, and thus will allow for reducing passenger uncertainty and its associated perceived cost, as well as to help passengers to time their arrival to the (skipped) stops and cut their otherwise waiting time.

\section{Case study}

A real bus line in the city of Dalian (China), line 26, is taken as a numerical example for applying the proposed model. Line 26, as shown in Figure 5, runs from Lingshui Passenger Transport Station to Wuyi Square, visiting 19 stops with $10.9 \mathrm{~km}$ in each direction, passing through a school zone (Dalian University of Technology, and the primary and high schools attached to Dalian University of Technology), a shopping center (Xian Road), and a business office zone (Software Park Service Center). a)

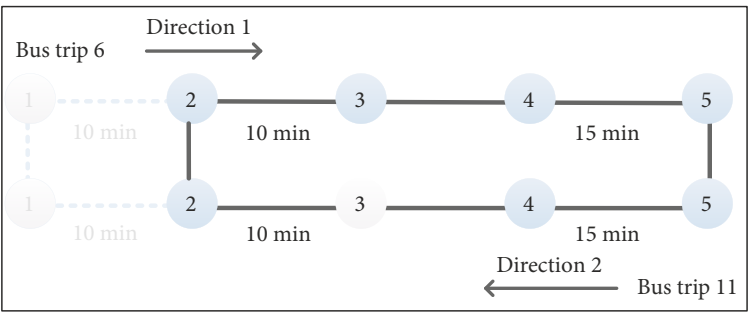

b)

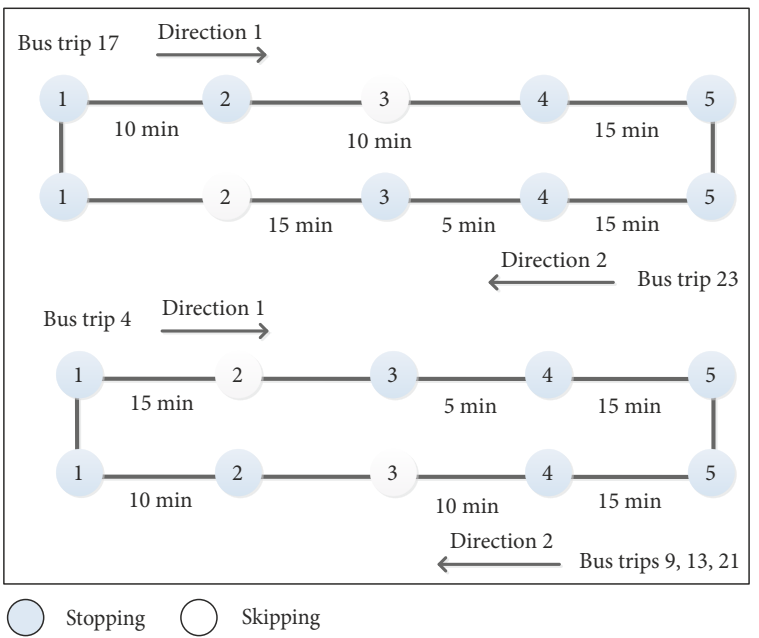

Figure 4. Optimal topologies of other strategies operated by bus trips: a - short turn strategy for trip 6 and mixed strategy for trip 11; b - limited stop strategy for trips 4, 9,13, 17, 21, 23

Table 3. Resulting timetable operated by FRO, limited stop, and mixed strategies

\begin{tabular}{|c|c|c|c|c|c|c|c|c|c|c|c|c|c|}
\hline & & \multicolumn{5}{|c|}{ Direction 1} & \multicolumn{5}{|c|}{ Direction 2} & \multirow[b]{2}{*}{$\begin{array}{l}\text { Bus } \\
\text { trip } \\
\text { No. }\end{array}$} & \multirow[b]{2}{*}{ Time period } \\
\hline \multicolumn{2}{|c|}{ Time } & 1 & 2 & 3 & 4 & 5 & 5 & 4 & 3 & 2 & 1 & & \\
\hline \multirow{7}{*}{$\begin{array}{l}7: 00 \ldots 8: 00 \\
\text { (at stop 2) }\end{array}$} & 1 & $7: 00$ & $7: 10$ & $7: 20$ & $7: 25$ & $7: 40$ & $7: 00$ & $7: 15$ & $7: 20$ & $7: 30$ & $7: 40$ & 8 & \multirow{6}{*}{$\begin{array}{l}7: 00 \ldots 8: 00 \\
\text { (at stop 3) }\end{array}$} \\
\hline & 2 & $7: 10$ & $7: 20$ & $7: 30$ & $7: 35$ & $7: 50$ & $7: 15$ & $7: 30$ & $* *$ & $7: 40$ & $7: 50$ & 9 & \\
\hline & 3 & $7: 25$ & $7: 35$ & $7: 45$ & $7: 50$ & $8: 05$ & $7: 20$ & $7: 35$ & $7: 40$ & $7: 50$ & $8: 00$ & 10 & \\
\hline & 4 & $7: 35$ & ** & $7: 50$ & $7: 55$ & $8: 10$ & $7: 25$ & $7: 40$ & ** & $7: 50$ & $* *$ & 11 & \\
\hline & 5 & $7: 40$ & $7: 50$ & $8: 00$ & $8: 05$ & $8: 20$ & $7: 30$ & $7: 45$ & $7: 50$ & $8: 00$ & $8: 10$ & 12 & \\
\hline & 6 & $* *$ & $7: 55$ & $8: 05$ & $8: 10$ & $8: 25$ & $7: 40$ & $7: 55$ & ** & $8: 05$ & $8: 15$ & 13 & \\
\hline & 7 & $7: 50$ & $8: 00$ & $8: 10$ & $8: 15$ & $8: 30$ & $7: 50$ & $8: 05$ & $8: 10$ & $8: 20$ & $8: 30$ & 19 & \multirow{6}{*}{$\begin{array}{c}\text { 8:00...9:00 } \\
\text { (at stop 3) }\end{array}$} \\
\hline \multirow{5}{*}{$\begin{array}{l}8: 00 \ldots 9: 00 \\
\text { (at stop 2) }\end{array}$} & 14 & $8: 00$ & $8: 10$ & $8: 20$ & $8: 25$ & $8: 40$ & $8: 05$ & $8: 20$ & $8: 25$ & $8: 35$ & $8: 45$ & 20 & \\
\hline & 15 & $8: 15$ & $8: 25$ & $8: 35$ & $8: 40$ & $8: 55$ & $8: 15$ & $8: 30$ & ** & $8: 40$ & $8: 50$ & 21 & \\
\hline & 16 & $8: 25$ & $8: 35$ & $8: 45$ & $8: 50$ & 9:05 & $8: 20$ & $8: 35$ & $8: 40$ & $8: 50$ & $9: 00$ & 22 & \\
\hline & 17 & $8: 40$ & $8: 50$ & $\star *$ & $9: 00$ & $9: 15$ & $8: 25$ & $8: 40$ & $8: 45$ & ** & $9: 00$ & 23 & \\
\hline & 18 & $8: 50$ & 9:00 & $9: 10$ & $9: 15$ & $9: 30$ & $8: 35$ & $8: 50$ & $8: 55$ & $9: 05$ & $9: 15$ & 24 & \\
\hline
\end{tabular}

Notes: (at stop2) or (at stop 3) representing the stop where the time period is determined; ${ }^{\star *}$ representing this time point is skipped by buses.

Table 4. Effects for passengers and operator introducing strategies

\begin{tabular}{|l|c|c|c|c|c|c|}
\cline { 2 - 7 } \multicolumn{1}{c|}{} & \multirow{2}{*}{$\begin{array}{c}\text { Total cost } \\
{[\$ / \text { hour] }}\end{array}$} & \multicolumn{2}{c|}{ Passenger costs [\$/hour] } & \multicolumn{2}{c|}{ Operator costs [\$/hour] } & Fleet size \\
\cline { 3 - 7 } & & Waiting time cost & In-vehicle time cost & Travel time cost & Empty seat hours cost & (buses) \\
\hline With strategies & 9674.67 & 1644.00 & 5755.67 & 2075.00 & 200.00 & 9 \\
\hline $\begin{array}{l}\text { Without } \\
\text { strategies }\end{array}$ & 9862.60 & 1476.44 & 5861.17 & 2200.00 & 325.00 & 10 \\
\hline \multirow{2}{*}{ Change } & -187.94 & 167.56 & -105.50 & -125.00 & -125.00 & -1 \\
\cline { 2 - 7 } & $-1.91 \%$ & $11.35 \%$ & $-1.80 \%$ & $-5.68 \%$ & $-38.46 \%$ & $-10 \%$ \\
\hline
\end{tabular}




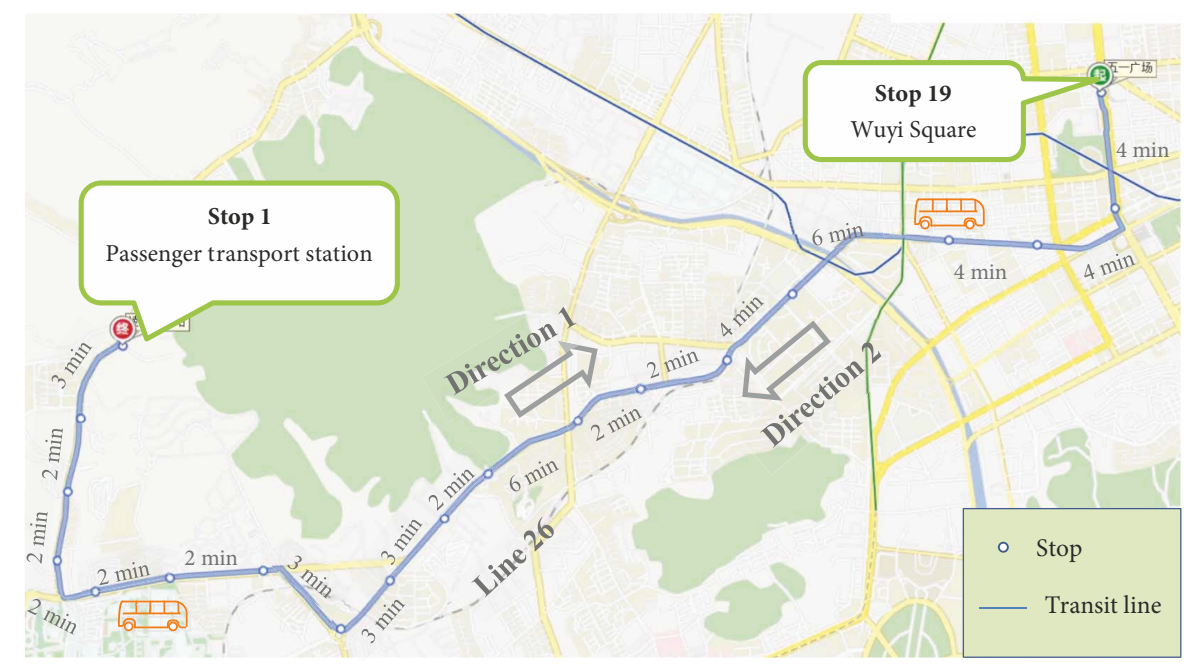

Figure 5. A real life transit route - line 26 in Dalian

In this case, a survey is conducted to collect data in the morning peak hours, 7:00...9:00, of the same day of week, including ridership, travel time between two consecutive stops, etc. Buses of line 26 use the isolated road during peak hours. The study period contains 19 bus trip departures in direction 1 and direction 2, respectively. The departure times from stop 1 in direction 1, Passenger Transport Station, are 7:00, 7:10, 7:20, 7:25, 7:30, 7:35, $7: 41,7: 45,7: 49$ 7:55, 8:00, 8:05, 8:10, 8:15, 8:20, 8:30, 8:40, 8:50 and 9:00, respectively. In the other direction, the departure times from stop 19, Wuyi Square, are 7:00, 7:10, 7:20, 7:30, 7:40, 7:45, 7:50, 7:55, 8:00, 8:05, 8:10, 8:15, 8:20, $8: 25,8: 30,8: 36,8: 45,8: 50$ and 9:00, respectively. The first operation period is 7:00 ..8:00, while 8:00 ..9:00 is for the second operation period. The departure times of these bus trips at the following stops are determined on the basis of travel times between two consecutive nodes illustrated in Figure 5. The desired occupancy of bus is set as 80 passengers. It will save 3 minutes for travel times if a bus skips a stop. Values of $15,10,150,2 \$$ /hour are used for the objective function weighting factors $c_{1}, c_{2}, c_{3}$ and $c_{4}$, respectively (Chen et al. 2015). Considering the real time bus arrivals available for passengers waiting at stops, the saving rate of waiting is set as 0.5 .

\subsection{Results}

The results obtained by the proposed methodology are shown in Figure 6. Bus trips have been scheduled to use four strategies: FRO, short-turn, limited stop, and mixed strategies during given operation periods in both directions. For instance, in direction 1 between 7:00...8:00 (Figure 6a), there are one bus trip operating short-turn strategy (e.g., departing from stop 3 at 7:40), one bus trip operating limited stop strategy (e.g., departing from stop 1 at 7:25), three bus trips operating mixed strategies (e.g., departing from stop 1 at 7:10, 7:45, 7:55) and six buses operating FRO strategies (e.g., departing from stop 1 at 7:00, 7:20, 7:30, 7:40, 7:49, 8:00). As is illustrated by Fig- ure 6, the use of a mixed strategy presents more saving of operator and users costs.

As shown in Table 5, shifting some FRO bus trips to use other strategies reduces the total costs of passengers and operator by $2.37 \%$, compared with all bus trips only operating FRO strategy. It may result in an increase of approximately $22.04 \%$ in passenger waiting time cost, while it also performs a reduction of around $3.00 \%$ in passenger in-vehicle time cost. Interestingly, there is greater savings for operator cost, such as saving $6.14 \%$ in bus travel time and $18.09 \%$ in empty seat hour costs. Additionally, it will save one bus, though this is not explicitly noted in the objective function. The promising results indicate that adjusting some FRO bus trips from a given timetable to operate by other strategies can be applied to real life public transit systems to improve operation efficiency, and improve the match between supply and demand.

\subsection{Sensitivity analysis for the saving time per a skipped stop}

Figure 7 illustrates the results of a sensitivity analysis of the cost components to changes of the saving time per a skipped stop. The parameter $t$ represents this saving time. It is found that the total cost, using the model with strategies, gradually decreases for $t$ increasing from 1 to 3 . Its greatest value is $26975.88 \$ /$ hour for $t=1$. However, it is below the total cost of using the model without strategies. That is, the total cost can be reduced by changing the operational strategies of some FRO bus trips. The larger is the saving per a skipped stop is, the less is the total cost. In addition, passenger cost, using the model with strategies, is more than that without the use of strategies; finally the operator cost is much lower with strategies than without the use of strategies. With the increase of $t$, passenger cost decreases and the operator cost increases because of the greater number of skipped stops resulting in saving invehicle time cost and increasing empty-seat hours cost. 
a)

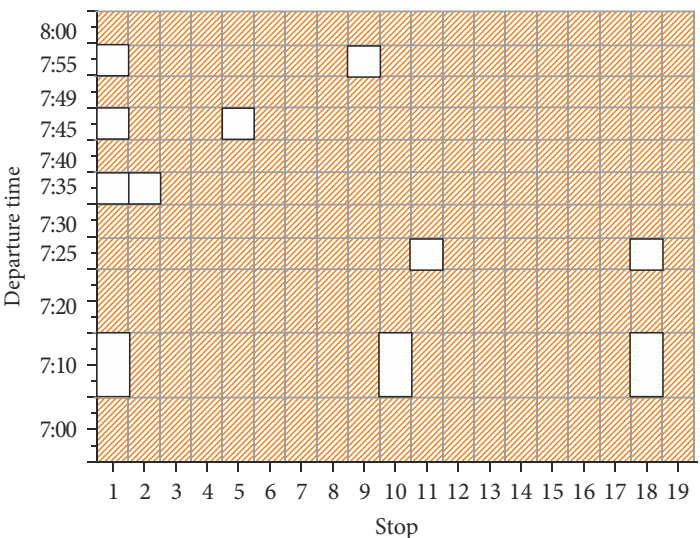

c)

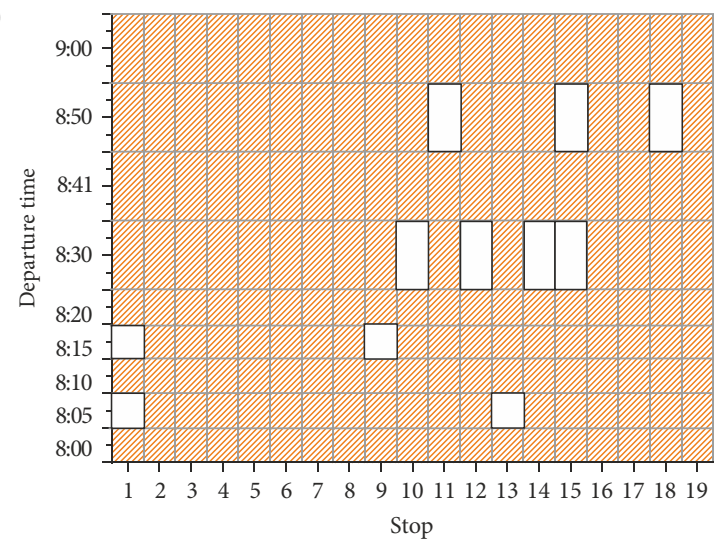

b)

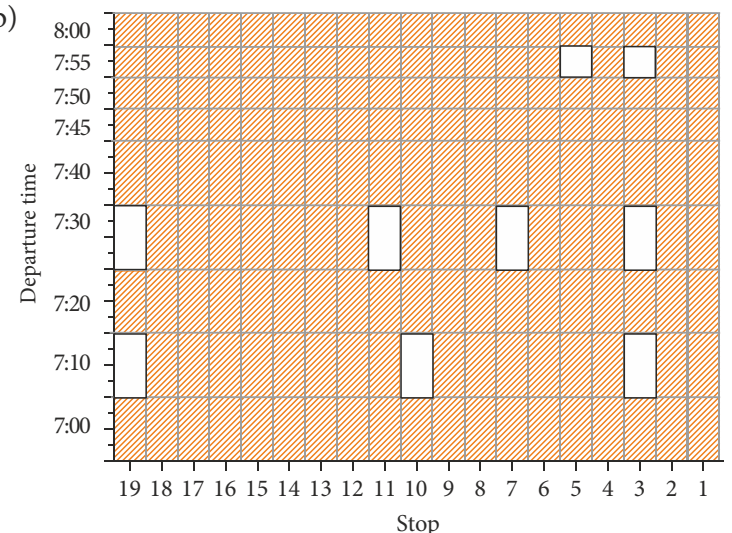

d)

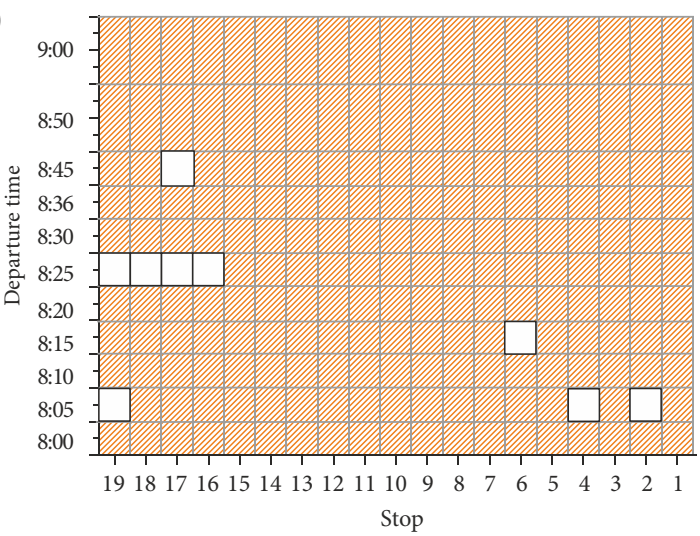

Figure 6. Optimal results with bus trips operating FRO, short turn, limited stop, and mixed strategies: a - 7:00...8:00 in direction 1; $\mathrm{b}-7: 00 \ldots 8: 00$ in direction $2 ; \mathrm{c}-8: 00 \ldots 9: 00$ in direction $1 ; \mathrm{d}-8: 00 \ldots 9: 00$ in direction 2

Table 5. Effects for passengers and operator introducing strategies

\begin{tabular}{|l|c|c|c|c|c|c|}
\cline { 3 - 6 } \multicolumn{1}{c|}{} & Total cost & \multicolumn{2}{|c|}{ Passenger costs $[\$ /$ hour $]$} & \multicolumn{2}{c|}{ Operator costs $[\$ /$ hour $]$} & \multirow{2}{*}{ Fleet size } \\
\cline { 3 - 6 } \multicolumn{1}{c|}{} & & Waiting time cost & In-vehicle time cost & Travel time cost & Empty seat hours cost & \\
\hline With strategies & 26742.98 & 2877.51 & 17722.59 & 4730.50 & 1412.39 & 22 \\
\hline Without strategies & 27392.28 & 2357.88 & 18270.10 & 5040.00 & 1724.31 & 21 \\
\hline \multirow{2}{*}{ Change } & -649.30 & 519.63 & -547.51 & -309.50 & -311.92 & -1 \\
\cline { 2 - 7 } & $-2.37 \%$ & $22.04 \%$ & $-3.00 \%$ & $-6.14 \%$ & $-18.09 \%$ & $-4.55 \%$ \\
\hline
\end{tabular}

a)

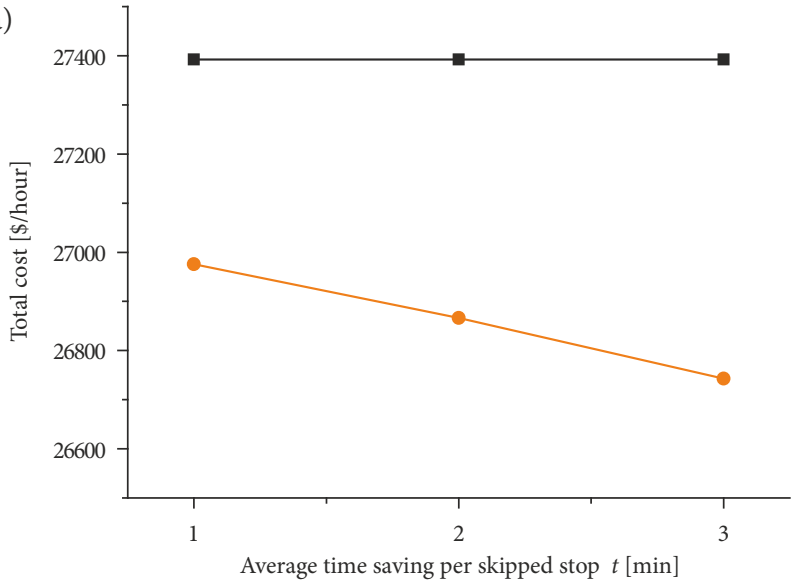

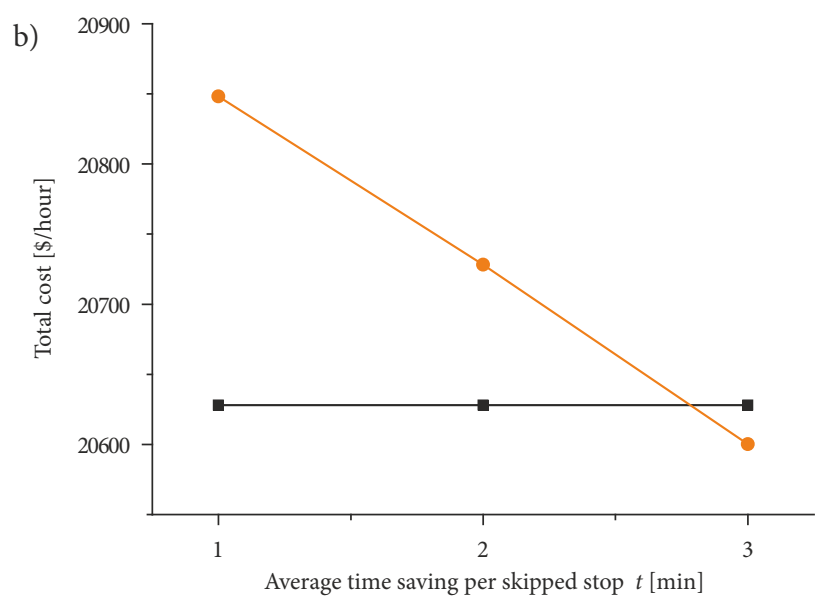

Figure 7 (to be continued) 


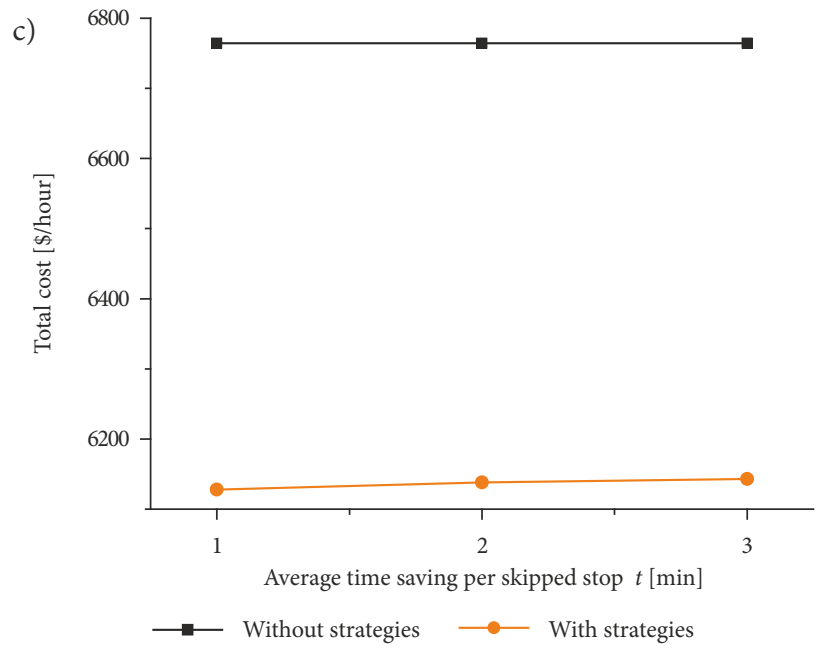

Figure 7. The effect of saving time per skipped stop parameter $t$ on optimal results: a - total costs; $\mathrm{b}$ - passenger costs; $\mathrm{c}$ - operator costs

\subsection{Computational times}

The results reported above are generated from a branch and bound algorithm coded in LINGO by a Dell computer with $2.2 \mathrm{GHz}$ Intel i5 CPU and $8 \mathrm{~GB}$ of RAM. The model is formulated as per the direction of travel for given operation period. There are 2947 variables and 4550 constraints for the optimization problem to generate the optimal results of Figure 6a. The execution time of the procedure varies between $30 \ldots 40$ minutes for the proposed model using the Dalian's case study.

\section{Conclusions}

This work has described the development of a methodology for an operational strategies problem based on a given timetable. Operational strategies consist of FRO, limited stop, short turn, and mixed strategy. This aims to better tailor supply to demand by shifting some FRO bus trips from a given timetable to use in accordance with the latter three strategies, and thus improve operation efficiency, while ensuring that the passenger load on each route segment does not exceed the desired occupancy. The proposed methodology has been applied to a numerical example and to a real life bus line in Dalian, China. The results show an indication that a significant saving could be attained by the use of multiple strategies. These savings were especially observed in the reduction of operational costs involved with the saving of travel times and running empty seats. That is, the use of other operational strategies proves more profitable with savings in the total costs for passengers and operators, compared with the current use of FRO strategy only. The amount of empty seat hours in public transport shows vehicle inefficiency. This is one of the most important operational efficiency measures. Interestingly, it demonstrates that the use of a combination of operational strategies can save empty seat hours. This is a significant saving indicating that the use of multiple strategies can reduce operation resources considerably.
Further studies can extend this proposed methodology to include multiple vehicle types. Multiple vehicle types investigated by Hassold and Ceder $(2012,2014)$, have demonstrated that operation efficiency can be improved by the reduction of vehicle load discrepancy. In this work, the results are promising, but further improvement in vehicle load discrepancy can be achieved by integrating operational strategies with the use of multiple vehicle types. Fleet size can be reduced by using the operational strategies, though this is not explicitly noted in this methodology. The graphic DF vehicle scheduling procedure by Ceder $(2007,2015)$, can be incorporated in this methodology to determine the number of vehicles required for covering all trips. Moreover, the effects of passenger boarding/alighting behaviours, stochastic vehicle arrivals (Chien et al. 2010b; Zhao, Chien 2015) and traffic conditions on the final results warrant further study.

\section{Acknowledgements}

The first author - Chunyan Tang - would like to acknowledge the support of the China Scholarship Council (CSC) for this study.

This work was supported in part by the National Natural Science Foundation of China (Grant No. 71801027) and partially by the Fundamental Research Funds for the Central Universities (Grant No. 3132018109, 3132016301).

\section{References}

Brooke, A.; Kendrick, D.; Meeraus, A.; Raman R. 2005. GAMS: $a$ User's Guide. GAMS Development Corporation.

Cats, O.; Loutos, G. 2016. Real-time bus arrival information system: an empirical evaluation, Journal of Intelligent Transportation Systems: Technology, Planning, and Operations 20(2): 138-151. https://doi.org/10.1080/15472450.2015.1011638

Ceder, A. 1989. Optimal design of transit short-turn trips, Transportation Research Record: Journal of the Transportation Research Board 1221: 8-22.

Ceder, A. 2015. Public Transit Planning and Operation: Modeling, Practice and Behavior. CRC Press. $742 \mathrm{p}$.

Ceder, A. 2007. Public Transit Planning and Operation: Theory, Modeling and Practice. Butterworth-Heinemann. 640 p.

Ceder, A.; Stern, H. I. 1981. Deficit function bus scheduling with deadheading trip insertions for fleet size reduction, Transportation Science 15(4): 338-363.

https://doi.org/10.1287/trsc.15.4.338

Chen, J.; Liu, Z.; Zhu, S.; Wang, W. 2015. Design of limited-stop bus service with capacity constraint and stochastic travel time, Transportation Research Part E: Logistics and Transportation Review 83:1-15. https://doi.org/10.1016/j.tre.2015.08.007

Chien, S.; Byun, J.; Bladikas, A. 2010a. Optimal stop spacing and headway of congested transit system considering realistic wait times, Transportation Planning and Technology 33(6): 495-513. https://doi.org/10.1080/03081060.2010.505048

Chien, S. I.-J.; Ulusoy, Y. Y.; Wei, C.-H. 2010b. Integrated transit services for minimum cost operation considering heterogeneous demand, Transportation Planning and Technology 33(7): 605-624. https://doi.org/10.1080/03081060.2010.512222 
Chiraphadhanakul, V.; Barnhart, C. 2013. Incremental bus service design: combining limited-stop and local bus services, Public Transport 5(1-2): 53-78. https://doi.org/10.1007/s12469-013-0067-7

Cortés, C. E.; Jara-Díaz, S.; Tirachini, A. 2011. Integrating short turning and deadheading in the optimization of transit services, Transportation Research Part A: Policy and Practice 45(5): 419-434. https://doi.org/10.1016/j.tra.2011.02.002

Delle Site, P.; Filippi, F. 1998. Service optimization for bus corridors with short-turn strategies and variable vehicle size, Transportation Research Part A: Policy and Practice 32(1): 19-38. https://doi.org/10.1016/S0965-8564(97)00016-5

Dziekan, K.; Kottenhoff, K. 2007. Dynamic at-stop real-time information displays for public transport: effects on customers, Transportation Research Part A: Policy and Practice 41(6): 489-501. https://doi.org/10.1016/j.tra.2006.11.006

Fu, L.; Liu, Q.; Calamai, P. 2003. Real-time optimization model for dynamic scheduling of transit Operations, Transportation Research Record: Journal of the Transportation Research Board 1857: 48-55. https://doi.org/10.3141/1857-06

Furth, P. G. 1987. Short turning on transit routes, Transportation Research Record: Journal of the Transportation Research Board 1108: 42-52.

Furth, P. G.; Day, F. B. 1985. Transit routing and scheduling strategies for heavy-demand corridors, Transportation Research Record: Journal of the Transportation Research Board 1101: 23-26.

Grossmann, I. E.; Viswanathan, J.; Vecchietti, A.; Raman, R. 2018. DICOPT. GAMS Development Corporation. Available from Internet: https://www.gams.com/latest/docs/S_DICOPT. html

Hassold, S.; Ceder, A. 2012. Multiobjective approach to creating bus timetables with multiple vehicle types, Transportation Research Record: Journal of the Transportation Research Board 2276: 56-62. https://doi.org/10.3141/2276-07

Hassold, S.; Ceder, A. 2014. Public transport vehicle scheduling featuring multiple vehicle types, Transportation Research Part B: Methodological 67: 129-143. https://doi.org/10.1016/j.trb.2014.04.009

Larrain, H.; Giesen, R., Muñoz, J. C. 2010. Choosing the right express services for bus corridor with capacity restrictions, Transportation Research Record: Journal of the Transportation Research Board 2197: 63-70. https://doi.org/10.3141/2197-08

Larrain, H.; Muñoz, J. C.; Giesen, R. 2015. Generation and design heuristics for zonal express services, Transportation Research Part E: Logistics and Transportation Review 79: 201212. https://doi.org/10.1016/j.tre.2015.04.008

Leiva, C.; Muñoz, J. C.; Giesen, R.; Larrain, H. 2010. Design of limited-stop services for an urban bus corridor with capacity constraints, Transportation Research Part B: Methodological 44(10): 1186-1201. https://doi.org/10.1016/j.trb.2010.01.003

Liu, Z.; Yan, Y.; Qu, X.; Zhang, Y. 2013. Bus stop-skipping scheme with random travel time, Transportation Research Part C: Emerging Technologies 35: 46-56. https://doi.org/10.1016/j.trc.2013.06.004

Qu, H.-Z.; Chien, S. I.-J.; Liu, X.-B.; Zhang, P.-T.; Bladikas, A. 2016. Optimizing bus services with variable directional and temporal demand using genetic algorithm, Journal of Central South University 23(7): 1786-1798. https://doi.org/10.1007/s11771-016-3232-8

Tang, C.; Ceder, A.; Zhao, S.; Ge, Y.-E. 2016. Determining optimal strategies for single-line bus operation by means of smartphone demand data, Transportation Research Record: Journal of the Transportation Research Board 2539: 130-139. https://doi.org/10.3141/2539-15

Ulusoy, Y. Y.; Chien, S. I.-J. 2015. Optimal bus service patterns and frequencies considering transfer demand elasticity with genetic algorithm, Transportation Planning and Technology 38(4): 409-424.

https://doi.org/10.1080/03081060.2015.1026101

Ulusoy, Y. Y.; Chien, S. I.-J.; Wei, C.-H. 2010. Optimal all-stop, short-turn, and express transit services under heterogeneous demand, Transportation Research Record: Journal of the Transportation Research Board 2197: 8-18.

https://doi.org/10.3141/2197-02

Viswanathan, J.; Grossmann, I. E. 1990. A combined penalty function and outer-approximation method for MINLP optimization, Computers \& Chemical Engineering 14(7):769-782. https://doi.org/10.1016/0098-1354(90)87085-4

Watkins, K. E.; Ferris, B.; Borning, A.; Rutherford, G. S.; Layton, D. 2011. Where is my bus? Impact of mobile real-time information on the perceived and actual wait time of transit riders, Transportation Research Part A: Policy and Practice 45(8): 839-848. https://doi.org/10.1016/j.tra.2011.06.010

Zhao, L.; Chien, S. I. 2015. Investigating the impact of stochastic vehicle arrivals to optimal stop spacing and headway for a feeder bus route, Journal of Advanced Transportation 49(3): 341-357. https://doi.org/10.1002/atr.1270 